\title{
Body Area Network for Ubiquitous Healthcare Applications: Theory and Implementation
}

\author{
Sana Ullah • Kyung Sup Kwak
}

Published online: 13 October 2011

(C) Springer Science+Business Media, LLC 2011

With recent advances in wireless communication, lowpower miniaturized sensors and semiconductor technologies, the sensor networks have become the integral part of ubiquitous healthcare systems. These networks can be used to provide remote health monitoring for a long period of time with real-time feedback to the hospital. For example, a set of intelligent and low-power sensors can be seamlessly integrated in/on a human body to create an autonomous sensor network called Body Area Network (BAN). The BAN can be used to stream biological information from the human body and transmit it over a long distance to a remote server for diagnostic recommendations. Some applications include diagnoses and treatment of many diseases including myocardial infarction, gastrointestinal tract, cancer detection, asthma, diabetes, and other health problems. Compared to traditional sensor networks, BANs face additional research challenges including signal propagation in/around a human body, power scavenging issues, fault tolerance, mobility, reliable Medium Access Control (MAC) protocols, Quality of Service (QoS), biocompatibility and security.

This special issue invited high quality unpublished research papers and review articles to disseminate the state-of-the-art research and development on BANs for ubiquitous healthcare systems, and to highlight open issues and challenges. Out of many good papers, eight papers were finally accepted for publication in this special issue. The summary of these papers is given below

S. Ullah $(\bowtie) \cdot$ K. S. Kwak

UWB-ITRC Center, Inha University,

Incheon, South Korea

e-mail: sanajcs@hotmail.com

S. Ullah

Chair of Pervasive and Mobile Computing, King Saud University,

Riyadh, Saudi Arabia
Jovanov et al. reviews the opportunities and challenges of BAN for ubiquitous healthcare applications. This paper provides a fundamental overview of BAN both for beginners and experts.

$\mathrm{Li}$ et al. presents the current status of IEEE 802.15.6 standardization where three PHYs and a single common MAC are discussed. In addition, the authors implements a prototype BAN system based on the high band of Ultrawide Band (UWB).

$\mathrm{Li}$ et al. proposes a novel MAC protocol for BAN called LDTAMAC, where the Guaranteed Time Slots (GTSs) are dynamically allocated according to the traffic load. To reduce the average packet delay the data packets are transmitted in the current superframe. In addition, the active portion of the superframe is adjusted in order to minimize the energy consumption. It is shown that the proposed protocol outperforms IEEE 802.15.4 in terms of packet delay and energy consumption.

Cheng et al. proposes another MAC protocol called RACOON. This protocol satisfies the inter-BAN QoS requirements and overcomes the performance degradation caused by mobility. Simulation results verify that RACOON has better QoS performance in terms of transmission latency, energy consumption, and user capacity compared with other QoS protocols.

Selimis et al. proposes a lightweight security scheme for BAN. This paper estimates the additional energy consumption introduced by several security schemes based on commercially available off-the-shelf hardware components (microprocessor and radio), the network topology and the MAC frame. Furthermore, the authors propose a new microcontroller design in order to reduce the energy consumption of the system. The new microcontroller needs fewer clock cycles and memory accesses to deliver the output. This microcontroller is optimized for the symmetric algorithm.

Chang et al. introduces a portable terrain adaptable fall detection system by placing accelerometers and gyroscopes 
on the human body and transmit data using wireless transmitter modules to mobile devices. This data is combined with the center of gravity clustering algorithm in order to compute the behavior patterns according to the relationship between the center of gravity and the feet portion of the body. This research work can aid future emergency call services or GPS positioning service to achieve immediate health attention to the fall victim.

Khan et al. proposes an interworking architecture for BAN within heterogeneous wireless networks using a cost function. The cost function is influenced by power consumption and data throughput. The simulation results show that the proposed scheme outperforms all other schemes in terms of throughput, delay and packet loss rate.

Chen et al. investigates the challenging issue of enabling fast brain-computer interaction to construct a mental speller. The authors explores the support vector machines-based single-trial estimation of evoked potentials. The results on a human subject show the advantages of the inducing paradigm used in the mental speller with a high classification rate.

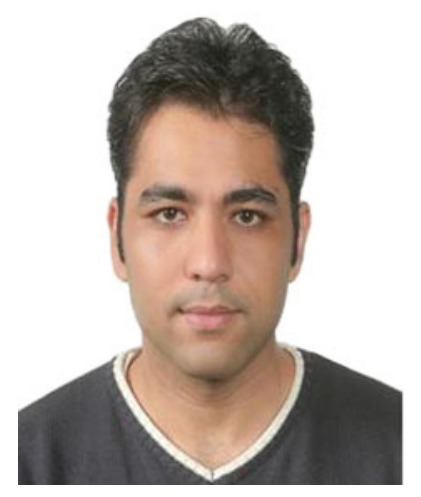

Sana Ullah received the Ph.D. degree in Information and Communication Engineering from Inha University in 2011. He received his M.S. in Computer Science and his B.S. in Mathematics, both from the University of Peshawar in 2006 and 2003 respectively. He has published more than 30 international journal and conference papers in Wireless Sensor and Wireless Body Area Networks. He has also contributed to IEEE 802.15.6 standard. $\mathrm{He}$ is in the editorial/reviewing committee of many international journals including IEEE Transaction on Wireless Communication, Computer Communications (Elsevier), EURASIP J. of Wireless Communication, and Journal of Medical System (Springer). His research interests are MAC protocol for Wireless Body Area Networks including Quality of Service
(QoS) and low-power implant communication, and crosslayer design. He is currently associated with the Chair of Pervasive and Mobile Computing at King Saud University, Saudi Arabia.

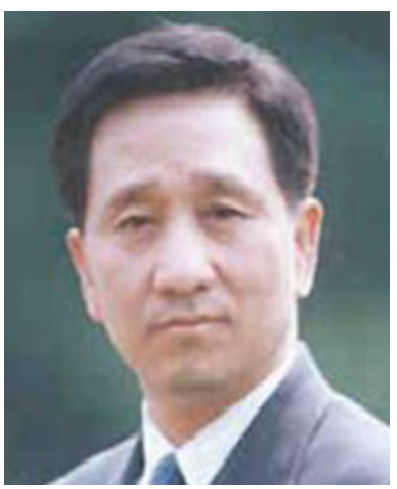

Kyung Sup Kwak received the B.S. degree from Inha University, Korea in 1977, and the M.S. degree from the University of Southern California in 1981 and the Ph.D. degree from the University of California at San Diego in 1988, respectively. From 1988 to 1989 he was a Member of Technical Staff at Hughes Network Systems, San Diego, California. From 1989 to 1990 he was with the IBM Network Analysis Center at Research Triangle Park, North Carolina. Since then he has been with the School of Information and Communication, Inha University, Korea as a professor. He had been the chairman of the School of Electrical and Computer Engineering from 1999 to 2000 and the dean of the Graduate School of Information Technology and Telecommunications from 2001 to 2002 at Inha University, Inchon, Korea. He is the current director of Advanced IT Research Center of Inha University, and UWB Wireless Communications Research Center, a key government IT research center, Korea. He has been the Korean Institute of Communication Sciences (KICS)'s president of 2006 year term. In 1993, he received Engineering College Young Investigator Achievement Award from Inha University, and a distinguished service medal from the Institute of Electronics Engineers of Korea (IEEK). In 1996 and 1999, he received distinguished service medals from the KICS. He received the Inha University Engineering Paper Award and the LG Paper Award in 1998, and Motorola Paper Award in 2000. His research interests include multiple access communication systems, mobile communication systems, UWB radio systems and ad-hoc networks, high-performance wireless Internet. 